\title{
The importance of graft kidney biopsy: A case of proteinuria one week after transplantation
}

Mariana Sousa ${ }^{1}$, Luís Falcão², Rita Calça ${ }^{3}$, Mário Gois ${ }^{4,5}$, Helena Viana ${ }^{4,5}$, Fernando Nolasco ${ }^{4,5}$

${ }^{1}$ Nephrology Department, Hospital Garcia de Orta

${ }^{2}$ Nephrology Department, Hospital Beatriz Ângelo

${ }^{3}$ Nephrology Department, Hospital Santa Cruz, Centro Hospitalar de Lisboa Ocidental

${ }^{4}$ Nephrology Department, Hospital de Curry Cabral, Centro Hospitalar Universitário de Lisboa Central

${ }^{5}$ Laboratory of Renal Morphology, Nephrology Department, Hospital Curry Cabral - Centro Hospitalar Universitário de Lisboa Central

\section{CLINICAL PRESENTATION}

We present a case of a 39-year-old African male with chronic kidney disease secondary to primary membranous nephropathy with positive anti-phospholipase $\mathrm{A} 2$ receptor autoantibodies (anti-PLA2R), in hemodialysis for one year. Past medical history included hypertension with hypertensive cardiomyopathy and dyslipidemia. The patient underwent a deceased-donor kidney transplant with three human leukocyte antigen (HLA) mismatches (two HLA class I and one HLA class II) with no donor-specific antibodies (DSAs) and cold ischemia of 8 hours. He had no history of previous kidney transplants. The residual diuresis was approximately $1000 \mathrm{~mL} / 24 \mathrm{~h}$. The titer of anti-PLA2R before transplantation was $139.40 \mathrm{RU} / \mathrm{mL}$ (negative $<20 \mathrm{RU} / \mathrm{mL}$ ).

Induction immunosuppression used was basiliximab (20 $\mathrm{mg}$ prior to transplant surgery, followed by a second dose of $20 \mathrm{mg} 4$ days after transplant) followed by tacrolimus, mycophenolate mofetil and prednisolone.

The early post-transplant period was uneventful, with an appropriate increase in urine output and decline in serum creatinine (nadir of $1.5 \mathrm{mg} / \mathrm{dL})$.

\section{Figure 1}

Periodic acid-Schiff (PAS) $\times 100$

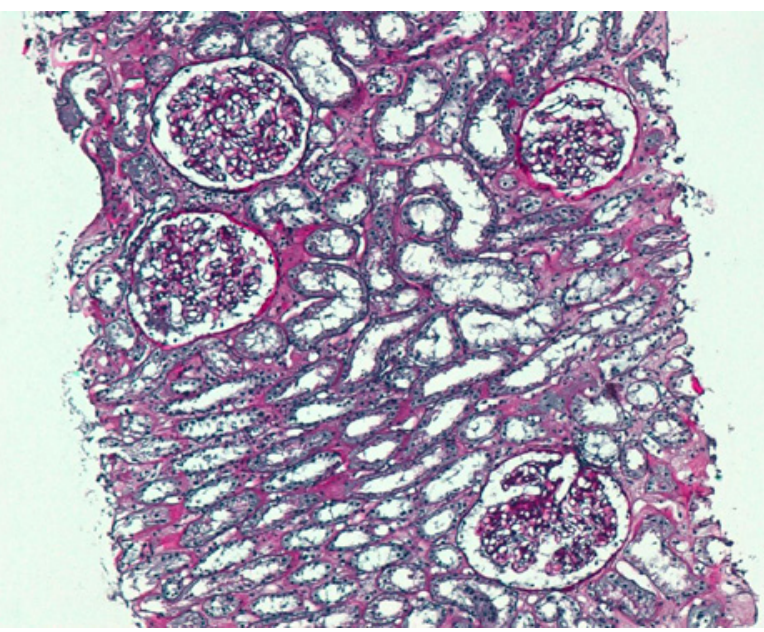

\section{Figure 2}

PAS $\times 400$

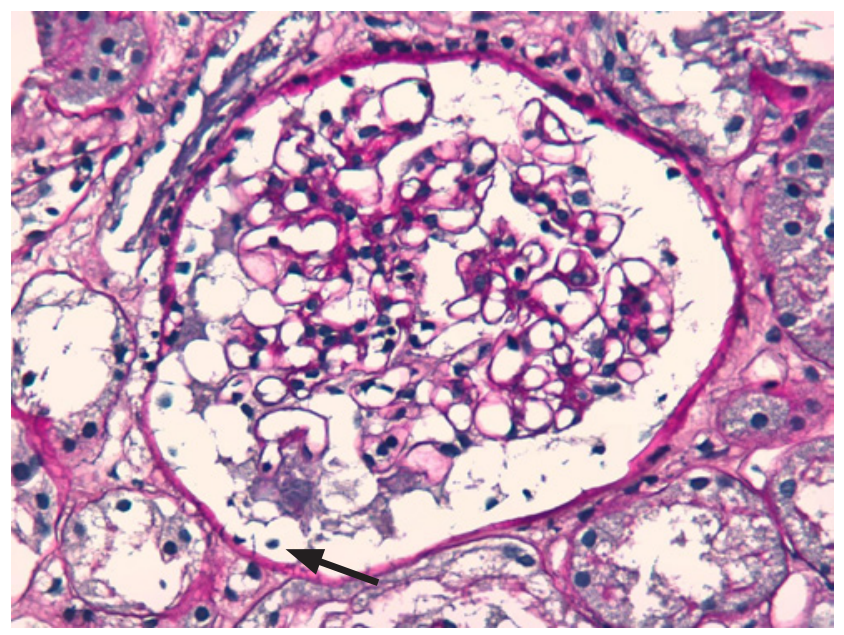

Figure 3

AntiPLA2R immunohistochemistry

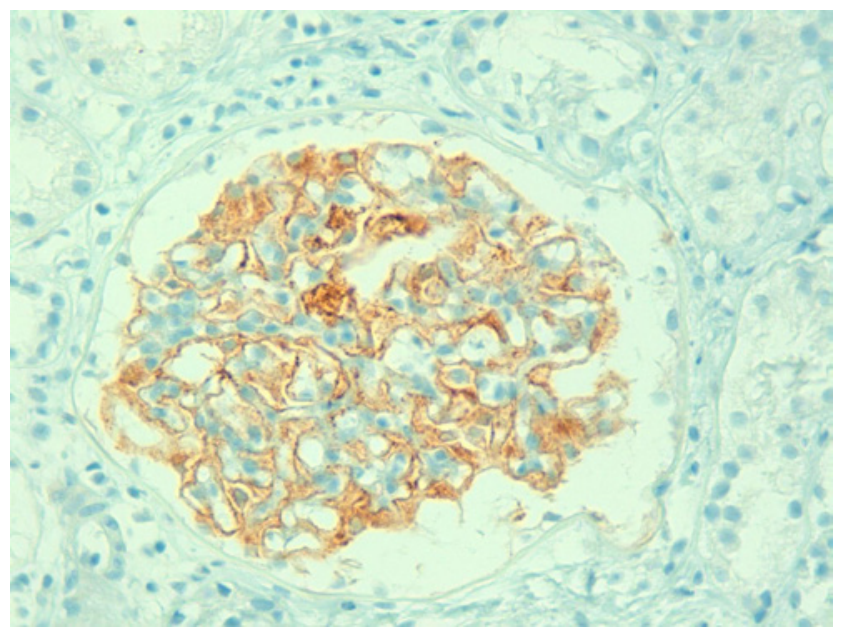




\section{Figure 4}

$\lg \mathrm{immunofluorescence}$

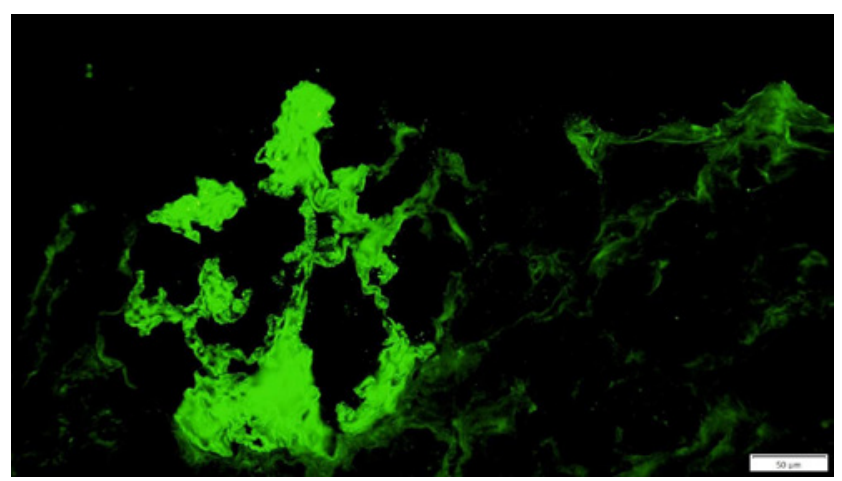

Eight days after transplant patient underwent a kidney graft biopsy because of 1.2 grams of proteinuria in a 24 -hour urine collection.

\section{QUESTIONS}

1. What is the most likely diagnosis, considering the clinical history and presentation?

2. What is the diagnosis considering light microscopy and immunofluorescence studies?

3. What is our patient's prognosis?

4. What is the role of anti-phospholipase $A 2$ receptor antibodies in pre-transplant evaluation and monitoring after transplantation?

5. What are the treatment options?

\section{ANSWERS}

1. What is the most likely diagnosis, considering the clinical history and presentation?

Proteinuria is commonly seen immediately after transplantation. At this early time point, it may be from the native kidneys or the allograft. This is a nonspecific finding that could represent a glomerular disease, including recurrent, de novo, and undefined glomerulonephritis or transplant-specific lesions (allograft nephropathy, transplant glomerulopathy, or acute rejection). ${ }^{1}$

In this case, the most likely diagnosis was an early recurrence of a membranous nephropathy (MN).

MN is a disease defined by IgG-containing subepithelial deposits in the glomerular capillary wall. The recent finding of a circulating antibody to the podocyte protein PLA2R cases of primary MN suggests in situ immune complex formation with this intrinsic autoantigen as the likely mechanism. ${ }^{2}$

MN can develop after renal transplantation and has a negative impact on graft survival.
The recurrence of MN has been described as "early recurrence" within the first 6-12 months after transplantation and a "late-onset recurrence" at about 5 years after transplant. ${ }^{3}$ The histologic features of early recurrence can be observed as early as 1-2 weeks after transplantation. ${ }^{4}$

This patient had two risk factors for recurrence: short waitlist time and high anti-PLA2R titer. Patients with these antibodies have a $60-70 \%$ risk of recurrence. ${ }^{5}$

Recently, potential predisposing factors were identified for recurrent $\mathrm{MN}$ including recipients who are older, recipient HLA-A3 antigen, steroid-free immunosuppressive regimen, and living related donor kidney. ${ }^{6}$ This patient had none of these risk factors.

Since the patient had residual diuresis, this grade of proteinuria could be from the native kidneys; however proteinuria levels before transplantation were not known.

The hypothesis of transplant-specific lesions is less probable as the patient has no immune risk factors and no decline in kidney function.

\section{What is the diagnosis considering light microscopy and immuno- fluorescence studies?}

The graft kidney biopsy consisted of subcapsular cortical fragment and contained 19 glomeruli and medium-sized arteries. In light microscopy (Figure 1 and 2), vacuolized and hypertrophic podocytes (arrow Figure 2) were evident with no other alterations of the glomeruli. A perivascular and periglomerular mononuclear inflammatory infiltrate involving $<10 \%$ of cortical was also visible. Arterioles were well preserved. C4d staining was negative in the peritubular capillaries, and there was no other evidence of acute rejection. In immunohistochemistry, a granular staining along the basement membrane of anti-PLA2R was detectable (Figure 3)

IgG positive diffuse granular deposits along the glomerular capillary wall were noticed in immunofluorescence (Figure 4). It was negative for other immunoglobulins, complement fragments, albumin and fibrin.

The diagnosis is a stage I membranous nephropathy.

3. What is the role of anti-phospholipase $A 2$ receptor antibodies in pre-transplant evaluation and monitoring after transplantation?

In patients with primary MN and positive anti-PLA2R monitoring, anti-PLA2R antibodies are useful in the follow-up and as prognostic marker. Serum anti-PLA2R antibody profiles reliably predict response to therapy, and levels at end of therapy may predict long-term outcome. ${ }^{7}$ Re-emergence of or increase in antibody titers precedes a clinical relapse. ${ }^{8}$

After kidney transplant, there is no consensus regarding the importance of anti-PLA2R baseline quantification when it comes to stratifying the risk of disease recurrence. 
Debiec et al. ${ }^{9}$ observed that some patients with anti-PLA2R1 antibodies at the time of transplantation will not develop recurrence (5 out of 10 patients).

On the other hand, the Mayo Clinic presented the largest cohort of patients with recurrent MN (33 patients) and found that patients with anti-PLA2R at the time of transplant had a higher risk of recurrence. ${ }^{10}$

Quintana et al. ${ }^{11}$ studied 21 Spanish patients with primary MN before transplantation. The recurrence of the disease was significantly related with anti-PLA2R seropositivity before transplantation. The authors found that an anti-PLA2R cut-off of $45 \mathrm{RU} / \mathrm{mL}$ pretransplantation was predictive of primary $\mathrm{MN}$ recurrence with a sensitivity of $85.3 \%$, and a specificity of $85.1 \%$.

In summary, the presence of anti-PLA2R antibody in the pretransplant period or at the time of transplantation seems to have clinical utility as a predicting risk factor for recurrent MN.

Patients with high anti-PLA2R levels or severe proteinuria in the pretransplant period should be carefully monitored after transplant, and a possible prophylactic therapy such as rituximab should be considered. In the posttransplant period, a vigilant monitoring of proteinuria and anti-PLA2R titers, as well as surveillance biopsies, would allow an earlier diagnosis of recurrent $\mathrm{MN}$, timely treatment and better outcomes.

\section{What are the treatment options?}

The patient was diagnosed with very early recurrent membranous nephropathy stage 1.

Even though the patient could benefit from standard supportive treatment such as renin-angiotensin system blockade and strict blood pressure control, the fact that the recurrence was in the very early posttransplant period meant these could compromise graft function.

Regardless of supporting treatment, most recurrent MN patients worsen ${ }^{3}$. Therefore, all patients should be considered for intensive treatment with immunosuppressive therapy if significant proteinuria is present $(>1 \mathrm{~g} / \mathrm{d})$. Spontaneous remission is uncommon in recurrent MN. ${ }^{12}$

In contrast to primary $\mathrm{MN}$, there is no evidence that steroids, alkylating agents, or calcineurin inhibitors provide specific benefits in recurrent MN since most transplant receivers who experience a recurrence are already receiving one or more of these agents. However, it is possible that, in selected cases, the standard transplant immunosuppressive regimen might be enough to induce immunological remission. 3,10

Overall, promising results with higher rates of clinical remission have been seen with rituximab but the side effects of rituximab, such as infections or the long-term risk of malignancy, have not been evaluated. ${ }^{10,12}$

This is a challenging case. Adding rituximab to maintenance therapy could increase the change of remission but with a great risk of infection, especially combined with high doses of the immunosuppressive drugs that patients need in this posttransplant period.
However, since the presentation was very early, calcineurin inhibitors and steroids could be enough to suppress anti-PLA2R formation and induce remission.

The patient started rituximab (two doses of $500 \mathrm{mg}$ ). At one-month follow up, proteinuria was $0.24 \mathrm{~g} / \mathrm{g}$ and the anti-PLA2R was negative.

\section{What is our patient's prognosis?}

Recurrent MN seems to have a more insidious course than primary $\mathrm{MN}$, with a potential histologic progression regardless of the clinical status that can lead to negative consequences on graft function. ${ }^{5}$

Grupper et al. ${ }^{10}$ reported a persistent histological activity in both untreated patients without progressive proteinuria, and in rituximab treated patients who obtained a complete or partial remission.

Protocol biopsies could be essential to attain a diagnosis before the progression of the disease to a more severe stage, when the disease may theoretically be more responsive to therapy. ${ }^{13}$

Disclosure of potential conflicts of interest: none declared

Authors' Contributions: Equal authorship of Mariana Sousa and Luís Falcão.

\section{References}

1. Shamseddin MK, Knoll GA. Posttransplantation proteinuria: An approach to diagnosis and management. Clin J Am Soc Nephrol. 2011;6(7):1786-1793.

2. Beck LH Jr, Bonegio RG, Lambeau G et al. M-type phospholipase A2 receptor as target antigen in idiopathic membranous nephropathy. N Engl J Med. 2009;361(1):11-21.

3. Dabade TS, Grande JP, Norby SM, Fervenza FC, Cosio FG. Recurrent idiopathic membranous nephropathy after kidney transplantation: A surveillance biopsy study. Am J Transplant. 2008;8(6):1318-1322.

4. Blosser CD, Ayalon R, Nair R, Thomas C, Beck LH. Very early recurrence of anti-phospholipase A2 receptor-positive membranous nephropathy after transplantation. Am J Transplant. 2012;12(6):1637-1642.

5. Passerini P, Malvica S, Tripodi F, Cerutti R, Messa P. Membranous nephropathy (Mn) recurrence after renal transplantation. Front Immunol. 2019;10(JUN):1-9.

6. Berchtold L, Letouzé E, Alexander MP, Canaud G, Logt A-E van de, Hamilton P, et al. HLA-D and PLA2R1 risk alleles associate with recurrent primary membranous nephropathy in kidney transplant recipients. Kidney Int [Internet]. 2020; Available from: https://doi.org/10.1016/j.kint.2020.08.007

7. Lee S. Anti-phospholipase A2 receptor antibodies in membranous nephropathy. Kidney Res Clin Pract. 2018;37(3):181-182.

8. De Vriese AS, Glassock RJ, Nath KA, Sethi S, Fervenza FC. A proposal for a serology-based approach to membranous nephropathy. J Am Soc Nephrol. 2017;28(2):421-430.

9. Debiec H, Martin L, Jouanneau C, Dautin G, Mesnard L, Rondeau E, et al. Autoantibodies specific for the phospholipase A2 receptor in recurrent and de novo membranous nephropathy. Am J Transplant. 2011;11(10):2144-2152.

10. Grupper A, Cornell LD, Fervenza FC, Beck LH, Lorenz E, Cosio FG. Recurrent membranous nephropathy after kidney transplantation: treatment and long-term implications. Transplantation. 2016;100(12):2710-2716.

11. Quintana LF, Blasco M, Seras M, Pérez NS, López-Hoyos M, Villarroel P, et al. Antiphospholipase $\mathrm{A} 2$ receptor antibody levels predict the risk of posttransplantation recurrence of membranous nephropathy. Transplantation. 2015;99(8):1709-1714.

12. Leon J, Pérez-Sáez MJ, Batal I, Beck LH, Rennke HG, Canaud G, et al. Membranous nephropathy posttransplantation: An update of the pathophysiology and management. Transplantation. 2019;103(10):1990-2002.

13. El-Zoghby ZM, Grande JP, Fraile MG, Norby SM, Fervenza FC, Cosio FG. Recurrent idiopathic membranous nephropathy: Early diagnosis by protocol biopsies and treatment with anti-CD20 monoclonal antibodies. Am J Transplant. 2009;9(12):2800-2807.

\section{Correspondence to:}

Mariana Moreira de Sousa, MD

Nephrology Department, Hospital Garcia de Orta, Almada, Portugal

Avenida Torrado da Silva, 2805-267 Almada

Email: sousamoreiramariana@gmail.com 